\title{
Effects induced by single and multiple dopants on the transport properties in zigzag-edged graphene nanoribbons
}

\author{
X. H. Zheng, ${ }^{1}$ I. Rungger, ${ }^{2}$ Z. Zeng, ${ }^{1, *}$ and S. Sanvito ${ }^{2}$ \\ ${ }^{1}$ Key Laboratory of Materials Physics, Institute of Solid State Physics, Chinese Academy of Sciences, Hefei 230031, China \\ ${ }^{2}$ School of Physics and CRANN, Trinity College, Dublin 2, Ireland
}

(Received 28 July 2009; revised manuscript received 12 October 2009; published 18 December 2009)

\begin{abstract}
The effects of boron and nitrogen doping on the transport properties of zigzag-edged graphene nanoribbons (ZGNRs) with antiferromagnetically coupled edge states are investigated by first-principles electronic structure combined with a nonequilibrium Green's function technique. Specifically, the effects produced by single and multiple impurities as a function of their distance from the edges are analyzed. It is found that the introduction of single $\mathrm{B}$ or $\mathrm{N}$ atoms induces bound states and quasibound states in ZGNRs, which can be observed as dips or peaks in the electron transmission function. In particular, the transmission channel associated to the edge states is strongly suppressed when the impurities are close to the edges. Multiple impurities in general interfere and modify further the transmission function. However, if the impurities are placed at positions such that the associated bound and quasibound states appear at opposite sides of the Fermi level, then the transmission can be rationalized as a simple superposition of the transmission function of individually doped ribbons. Finally, an interesting situation appears for B and $\mathrm{N}$ codoping, since fully spin-polarized transmission peaks are generated at energies corresponding to the ribbon gap. This offers the hope of using such nanoribbons for low-bias spin-polarized tunneling in spintronics applications.
\end{abstract}

DOI: 10.1103/PhysRevB.80.235426

PACS number(s): 73.63.-b, 85.65.+h, 72.25.-b

\section{INTRODUCTION}

Graphene nanoribbons (GNRs) have attracted great interests in recent years due to their remarkable structural and electronic properties and their appeal for potential applications in nanoelectronic devices. ${ }^{1-11}$ The peculiarity of GNRs resides in the fact that their characteristics depend strongly on their size and on the shape of their edges. ${ }^{10-12}$ By controlling the size and edge shape, one can obtain metals or semiconductors with an energy gap that depends on the ribbon width. Thus, GNRs can provide a multitude of building blocks for the construction of nanoelectronic devices. More interestingly, in the zigzag-edged GNRs (ZGNRs) the unpaired $\pi$ and $\pi^{*}$ electrons strongly localized at the ribbons edges order magnetically. In the ground state these edge states are spin-degenerate but spatially separated, i.e., edge states with different spins are localized on different sides of the ribbon. It is predicted that there are two stable spin configurations: ferromagnetic (FM) and antiferromagnetic (AF) ${ }^{5,10,13} \mathrm{FM}$ or AF here means that the spins of the two edge states are either parallel or antiparallel to each other. First principles calculations show that ZGNRs are metallic in the FM configuration, while they are semiconducting in the $\mathrm{AF}$ one, and that the $\mathrm{AF}$ state has lower energy. Due to their magnetism, ZGNRs are believed to be promising materials for spintronic devices.

In general, the properties of GNRs are very sensitive to many factors, such as doping, defects, edge modification, molecule adsorption and external electrical field. These provide plenty of opportunities for tuning and greatly enlarge the applications of GNRs. For example, AF-ZGNRs are semiconductors, but they can be tuned to be half-metallic by applying an external electrical field, by edge decoration or by B-N chain substitution. ${ }^{5,14-16}$ This paves the way for new possible applications in spintronics, since completely spin polarized transport can be realized. Among all the possibilities, impurity doping is one of the most frequently adopted ways for tuning the properties of GNRs. Another example provided by Martins shows that $\mathrm{B}$ doping can give rise to spin-polarized transmittance in ZGNRs with FM order. ${ }^{13} \mathrm{Up}$ to now, most of the doping effect studies in ZGNRs focus on magnetism or spin polarized transport.

In this work, the changes in the electronic structure induced by $\mathrm{B} / \mathrm{N}$ doping and the associated effects on the transport properties of ZGNRs have been studied by firstprinciples calculations. Since the most stable state of ZGNRs is AF, we will only discuss AF-ZGNRs. In particular, the dependence of the various effects on the specific doping site is systematically investigated. We find that both $\mathrm{B}$ and $\mathrm{N}$ atoms can induce quasibound states and bound states in GNRs, which are localized both in energy and in space. In an ideal transport experiment, while quasibound states are coupled to the propagating states of the electrodes, bound states are truly localized, and decay exponentially away from the impurity site. Quasibound states locate far from the gap and result in transmission dips, while bound states appear in the energy gap and give rise to transmission peaks when their coupling to the leads is turned on by disorder. As a result of the formation of quasibound states, the density of states (DOS) of the edges states decreases toward the impurity atom and becomes negligible at the impurity atom site itself. Thus the electron transmission through the edge states is greatly suppressed.

The rest of this paper is organized as follows: in Sec. II, we give a brief description of the geometry used for the ZGNRs and of the computational method, while in Sec. III, the main results are presented and discussed. Finally in Sec. IV, we draw out conclusions. 
(a)

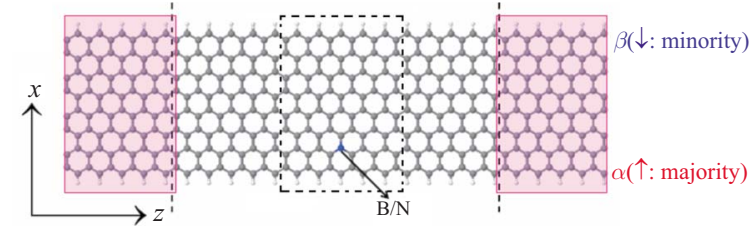

(b)

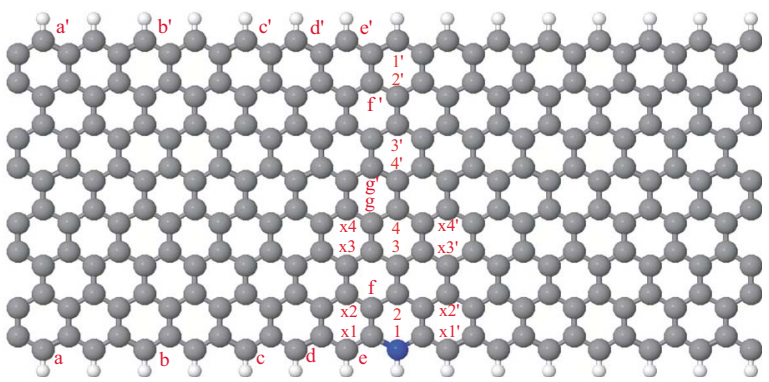

FIG. 1. (Color online) (a) Structure of the two-probe device made of an 8-ZGNR. The shadowed areas indicate the leads unit cell. The scattering region between the electrodes contains 3 of such cells. $\alpha$ (spin majority) and $\beta$ (spin minority) are the spin indices and label the antiferromagnetically aligned spin-polarized edges. In (b) we define the indices describing the impurities positions in the scattering region. Thus $1,2, \ldots, 1^{\prime}, 2^{\prime}, \ldots$, and $\mathrm{x} 1, \mathrm{x} 1^{\prime}, \ldots$ (in red) indicate the possible doping sites. The labels $a, b, \ldots$ and $a^{\prime}, b^{\prime}, \ldots$ are for the atoms used in the calculation of the projected density of states. The atom shown in blue is one of the doping impurity and it is shown just as illustration.

\section{CALCULATION DETAILS}

The model structure for a two terminal device is constructed as follows: the scattering region is seamlessly connected to two semi-infinite zigzag $(8,0)$ graphene nanoribbons of the same width [following a previous convention, this is called 8-ZGNR (Refs. 5 and 10)]. The edge $\mathrm{C}$ atoms are terminated by $\mathrm{H}$. The leads unit cell contains 90 atoms, and it has a total length of $12.3 \AA$ along the $z$ direction [Fig. 1(a)]. The scattering region consists of three lead unit cells with a total length of $36.9 \AA$ and includes 270 atoms. In order to investigate the effects of impurities on the transport properties, one or several $\mathrm{C}$ atoms are replaced by $\mathrm{B}$ or $\mathrm{N}$ in the scattering region [see Fig. 1(a)]. We systematically study the effects of the doping as a function of the doping site. This is defined by its distance from the zigzag edges. For single impurity, the $\mathrm{B}$ or $\mathrm{N}$ atoms are located at the center of the scattering region along the $z$ direction. Due to the structural symmetry, we only consider doping at sites 1, 2, 3, and 4, which are close to the lower edge [see Fig. 1(b) for the definition of the different sites]. For multiple impurities, we investigate several cases, namely: (i) two impurity atoms placed at equal distance from the lower edge of the ZGNR, (ii) two atoms placed at an equal distance from the two opposite edges (e.g., 1 and $1^{\prime}, 2$ and $2^{\prime}$, etc.), (iii) the first impurity placed at the edge and the second one inside the ribbon. Since in ZGNRs the valence and conduction edges states of two spin channels are energy degenerate but spatially separated, we define the valence edge state at the lower side as spin majority $(\alpha)$ and the valence edge states at the upper side as spin minority $(\beta)$ [see Fig. 1(a)].
All calculations are performed with an ab initio scheme combining density functional theory (DFT) with the nonequilibrium Green's functions (NEGF) method for quantum transport. The electronic structure of the leads is computed with the SIESTA package, ${ }^{17}$ which employs normconserving pseudopotentials and linear combinations of atomic orbitals as basis set. The wave function is expanded over a single zeta basis set to get a reasonable balance between computation effort and accuracy. Our tests comparing a single zeta and a double zeta plus polarization bases show little difference in the band structure. This choice was also adopted in the transport calculations of carbon systems by other authors. ${ }^{18,19}$ The resolution of the real space grid is determined by an equivalent plane wave cutoff of $200 \mathrm{Ry}$. The exchange-correlation potential is at the LDA level in the form of Perdew-Zunger. ${ }^{20}$

Transport calculations are performed with the SMEAGOL code ${ }^{21-23}$ which interfaces SIESTA and calculates selfconsistently the density matrix of the scattering region in the presence of an external bias by means of the NEGF technique. The bias is applied by setting the chemical potential of the left and right leads to $\mu_{L}=\mu+e V_{b} / 2$ and $\mu_{R}=\mu-e V_{b} / 2$, respectively, where $\mu$ is the common Fermi level of both leads and $V_{b}$ is the applied bias. The transmission function is calculated using the Landauer formula ${ }^{24,25}$

$$
T\left(E, V_{b}\right)=\operatorname{Tr}\left[\Gamma_{L}\left(E, V_{b}\right) G^{R}(E) \Gamma_{R}\left(E, V_{b}\right) G^{A}(E)\right],
$$

where $G^{R / A}$ are the retarded/advanced Green's functions of the scattering region and $\Gamma_{L / R}$ are coupling matrices to the left/right leads. In this work, we only consider the zero bias case, namely, $V_{b}=0$.

Perfect ZGNRs are semiconducting and there is no coupling between the scattering region and the leads for energies within the gap around the Fermi energy $\left(E_{F}\right)$. However, in real physical situations it is very unlikely to prepare a GNR with an ideally periodic structure, since disorder of various origin always exists. This will introduce a finite DOS in the band gap and therefore also a finite coupling between the scattering region and the leads even at these energies. In this work, generic disorder in the leads is taken into account in an approximate way by adding a finite imaginary part $\delta$ to the energy in the calculation of self energies. ${ }^{23}$

\section{RESULTS AND DISCUSSIONS}

\section{A. Single dopant: Transmission}

The transmission function for perfect leads $(\delta=0)$ and single B doping under different doping conditions is shown in Fig. 2. When doping $B$ at site 1 (at the ribbon edge, the position with the lowest energy) transmission dips in both spin channels can be observed at around $0.6 \mathrm{eV}$ (above $E_{F}$ ) [see Fig. 2(a)]. At the top of the valence band (VB) the transmission of the majority spins drops from 2 to $1 \mathrm{G}_{0}$ $\left(\mathrm{G}_{0}=e^{2} / h\right.$ : transmission quantum for one spin channel). In contrast for the minority spins transmission at the top of the VB remains almost unchanged as compared to the undoped case. Opposite is the case at the bottom of the conduction band $(\mathrm{CB})$ : here the majority spin transmission is barely af- 

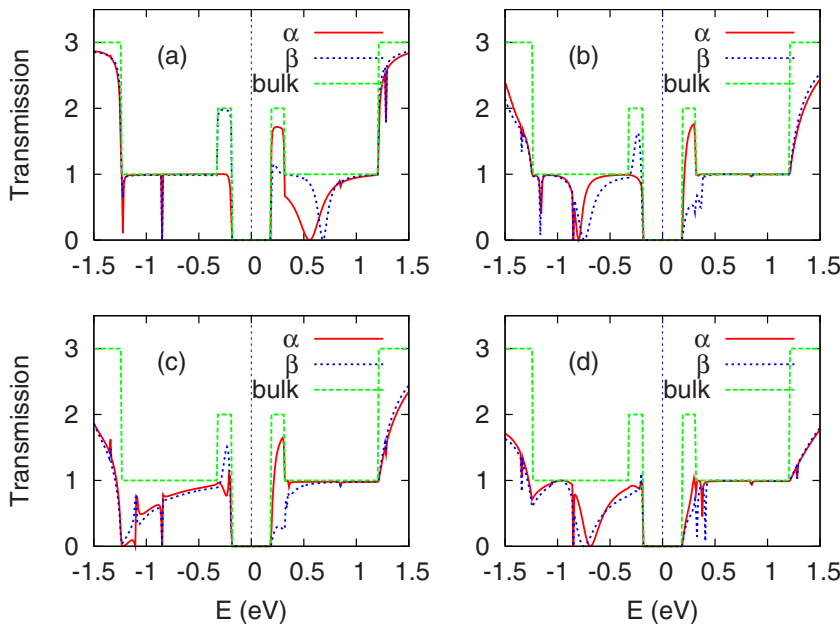

FIG. 2. (Color online) The transmission function for the ribbon doped with B at different positions: (a) site 1, (b) site 2, (c) site 3, and (d) site 4. See Fig. 1 for the definition of the different sites. For comparison, the transmission function for the undoped system is also shown (due to spin degeneracy only one spin is shown).

fected by the doping, whereas the minority spin transmission is approximately halved.

When doping $\mathrm{B}$ at site 2, transmission dips can be observed at $E-E_{F}=-0.8 \mathrm{eV}$ [see Fig. 2(b)]. By comparison with doping at site 1 [Fig. 2(a)] in the case of site 2 the transmission close to the top of the VB and close to the bottom of the $\mathrm{CB}$ is now reduced in both the spin channels. This effect is further enhanced when doping at site 3 [Fig. 2(c)], and gets finally maximized when the B sits in the middle of the ribbon. In this case the transmission is smaller than 1 for both spin channels at both band edges [Fig. 2(d)]. In summary, as we move the $\mathrm{B}$ atom from the edges to the center, the transmission through both spins is progressively suppressed. Our results confirm a very recent work by Biel et al., who also reported that a B impurity can present either donor or acceptor character depending on its actual position with respect to the ribbon edge. ${ }^{26}$

Note that in all the cases investigated, the breaking of the ribbon mirror symmetry has different effects on the edge states at the two sides of the ribbon, the spin degeneracy is broken, and the transmission function becomes spin dependent. For $\mathrm{N}$ doping, similar results are obtained. The main difference with $\mathrm{B}$ is that the dips in the transmission function introduced by $\mathrm{N}$ are always opposite in energy with respect to $E_{F}$ to those generated by $\mathrm{B}$ (see Fig. 3). For instance doping $\mathrm{N}$ at site 1 produces a dip in the VB and leaves the $\mathrm{CB}$ practically unchanged. Similar features have been already reported for $\mathrm{B}$ and $\mathrm{N}$ doping in metallic carbon nanotubes. ${ }^{18,27}$

When perfect leads are used the transmission in the gap is exactly zero [see Figs. 2 and 3]. We now take into account generic disorder in the leads by adding an artificial broadening $\delta=1.0 \times 10^{-4}$ Ry to the energy entering the self-energies. In this way the DOS of the leads is nonzero at all energies, ${ }^{23}$ so that we can turn on the coupling between the scattering region and leads also for states placed in the gap. We take B doping as an example and show in Fig. 4 the resulting trans-
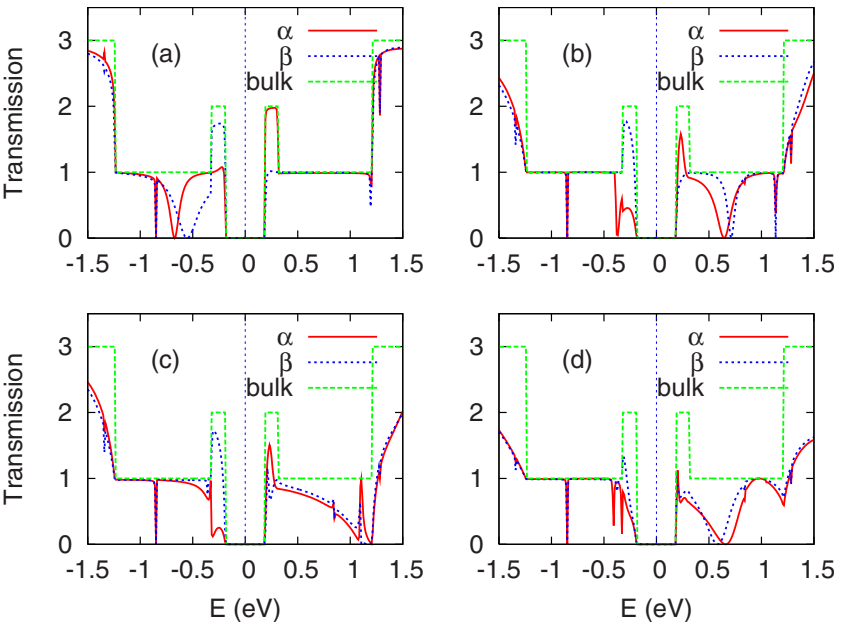

FIG. 3. (Color online) The transmission function for the ribbon doped with $\mathrm{N}$ at different positions: (a) site 1, (b) site 2, (c) site 3, and (d) site 4. See Fig. 1 for the definition of the different sites. For comparison, the transmission function for the undoped system is also shown (due to spin degeneracy only one spin is shown).

mission function, this time plotted on a logarithmic scale. Again we present results for different doping sites, but in the smaller energy range $[-0.3,0.3] \mathrm{eV}$, which spans the gap. We observe that there is almost no change in transmission for energies outside the gap. In contrast and as expected, inside the gap the transmission is now finite, although rather small. The actual magnitude depends on the value used for $\delta,{ }^{23}$ where a large $\delta$ represents large disorder. We observe that a few sharp transmission peaks appear in the gap as a result of the introduction of the impurity atoms (see Fig. 4). Note that, although the broadening of the bound states is artificially governed by $\delta$, their position is a feature of the electronic structure and remains unchanged for broadening down to $10^{-7}$ Ry. For $\mathrm{N}$ doping an analogous behavior is found, with the difference that, while for B doping the bound states are
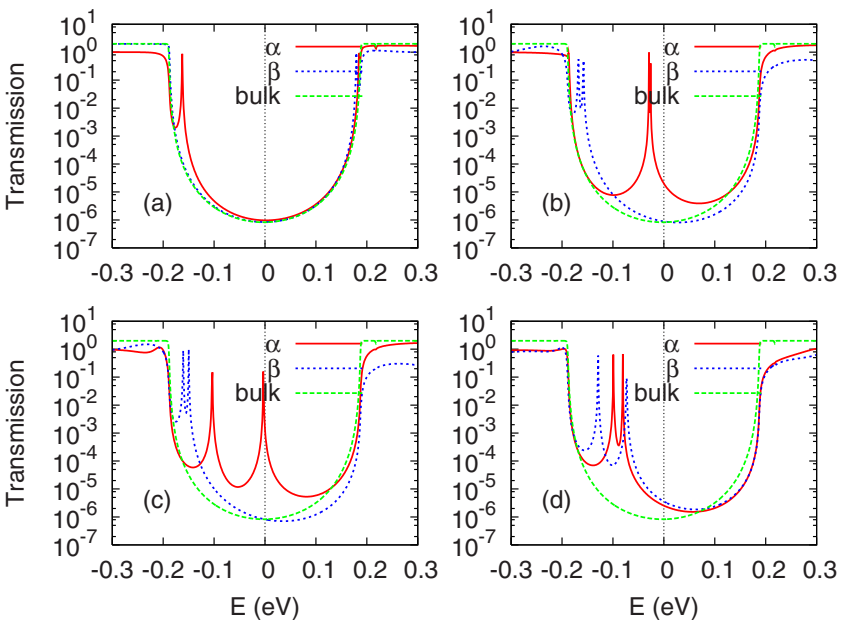

FIG. 4. (Color online) The transmission function plotted in the energy range $[-0.3,0.3] \mathrm{eV}$ for the same $\mathrm{B}$ doping sites as shown in Fig. 2. This time the leads electronic structure has been broadened artificially by adding a small imaginary energy $\delta=10^{-4} \mathrm{Ry}$, in the construction of the leads self-energies. 

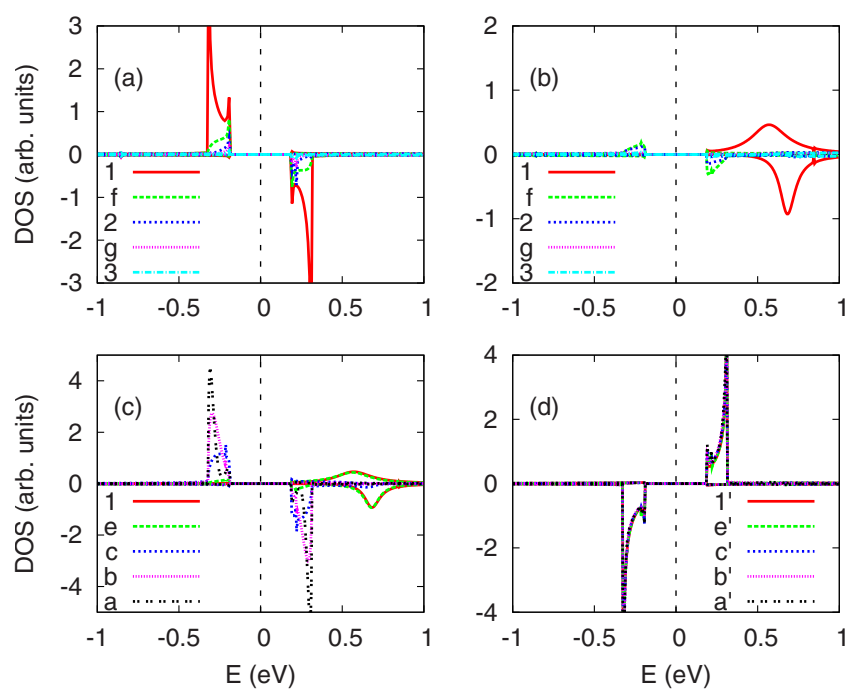

FIG. 5. (Color online) PDOS for different atomic sites and species of variously doped nanoribbons. (a) PDOS of $\mathrm{C}$ atoms located at different positions with increasing distance from the lower edge of the undoped ribbon. PDOS for $\mathrm{C}$ atoms located at different positions with respect to a single B dopant: (b) for atoms with increasing distance from $\mathrm{B}$ in the $x$ direction, when $\mathrm{B}$ is at the position 1; (c) for atoms with increasing distance from a central B atom placed on the lower edge; (d) for atoms with increasing distance to central atom B placed on the upper edge. As a convention, positive values of PDOS are for the majority spins and negative values for the minority spins.

always below the Fermi level, for $\mathrm{N}$ doping they are always above it.

\section{B. Single dopant: Electronic structure}

We now investigate the origin of the three main features in the transmission spectrum: (1) transmission dips in the energy regions $[-1.0,-0.5] \mathrm{eV}$ and $[0.5,1.0] \mathrm{eV}$; (2) transmission peaks in the energy gap $[-0.19,0.19] \mathrm{eV}$ when $\delta$ $=10^{-4} \mathrm{Ry}$; (3) transmission suppression at the top of the VB and at the bottom of the CB. To this aim we have calculated the projected density of states (PDOS) of the carbon atoms, focusing mainly on the edge carbon atoms and on the atoms surrounding the impurity. We take B doping at site 1 and site 2 as an example.

In the energy ranges $[-0.32,-0.19]$ and $[0.19,0.32] \mathrm{eV}$ the DOS of the undoped system is dominated by contributions from the edge carbon atoms [see Fig. 5(a)]. These are the well-known edge states, and their density of states decays rapidly from the edge toward the center of the ribbon [see Fig. 5(a)]. Due to the edge states at the top of the VB and at the bottom of the $\mathrm{CB}$ the transmission is $T=2$, while below and above them it is only $T=1$ [Fig. 2]. Therefore, the band belonging to the edge states provides an additional transmission channel in this energy range.

When one edge carbon atom (position 1) is substituted by $\mathrm{B}$, the DOS of the edge states is significantly modified by the interaction with the impurity. In the energy range $[-0.32$, $-0.19]$ and $[0.19,0.32] \mathrm{eV}$ the contribution of atom " 1 " (the $\mathrm{B}$ doping site) is approximately zero, and the PDOS of that
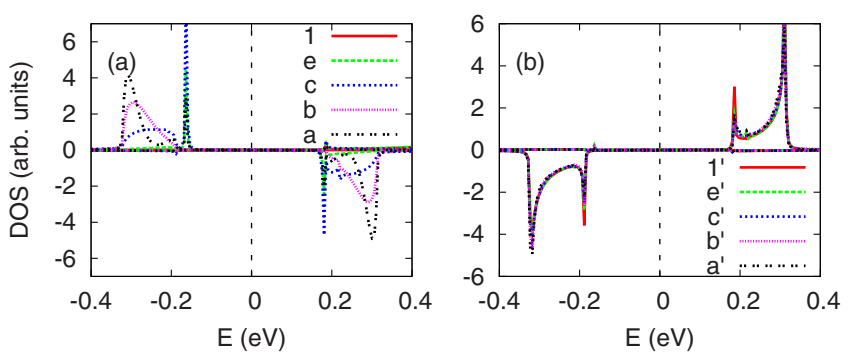

FIG. 6. (Color online) PDOS for atoms located at different sites when one $\mathrm{B}$ atom is doped at site 1 and calculated with $\delta$ $=10^{-4} \mathrm{Ry}$ : (a) for atoms with increasing distance to a central $\mathrm{B}$ on the lower edge; (b) for atoms with increasing distance to central B on the upper edge.

site is mainly concentrated in the interval $[0.6,0.7] \mathrm{eV}$ [see Fig. 5(b)]. We note that the PDOS is calculated by using the NEGF so that the energy broadening of a given state is given entirely by its coupling to the leads (no additional broadening is added at this stage). By looking at the lower edge carbons, we find that in the energy range $[-0.32$, -0.19] eV the PDOS decays almost exponentially from the two ends of the scattering region toward the $\mathrm{B}$ doping site [see Fig. 5(c)]. The presence of the B breaks the extended nature of the lower edge resulting in the reduction of the transmission function of the majority spins from 2 to 1 . This means that a single impurity placed over the ribbon edge can suppress completely the transmission through the associated edge state. This effect mirrors that on the local magnetism, which can also be suppressed by a single impurity or a defect placed over the ribbon edge. ${ }^{28}$ In contrast, the edge state located at the upper edge is not affected [see Fig. 5(d)], thus the spin minority transmission at the top of the VB is still approximately 2 [see Fig. 2(a)]. The opposite situation is found at the bottom of the $\mathrm{CB}$ : here the transmission through edge states is suppressed for the minority spin, which are localized on the lower edge, whereas it remains unchanged for the majority spin localized on the upper edge in this energy range.

The atoms around the $\mathrm{B}$ atom contribute significantly to the DOS peaks around [0.6,0.7] eV [see Fig. 5(b)] for both spins. These broad peaks in the DOS correspond to quasibound states, which are localized around B and decay away from the impurity center. The large energy broadening of these peaks indicates a strong coupling to the leads. These quasibound states produce an enhanced backscattering of the electrons, so that transmission dips are observed in this energy range [see Fig. 2].

We now analyze the effects introduced by disorder in the leads. Disorder is mimicked by adding an imaginary part $\delta$ $=10^{-4}$ Ry to the energy during the construction of the leads self-energies needed for calculating the Green's function of the scattering region. The resulting PDOS for an energy range around the band gap is shown in Fig. 6. Outside the gap the PDOS is basically identical to the one obtained for $\delta=0$ [Figs. 5(c) and 5(d)]. However inside the gap (in the range $[-0.19,0.19] \mathrm{eV})$ we observe very narrow peaks in the DOS (at $-0.16 \mathrm{eV}$ for the majority and at $0.18 \mathrm{eV}$ for the minority spin). These states are extremely localized around 

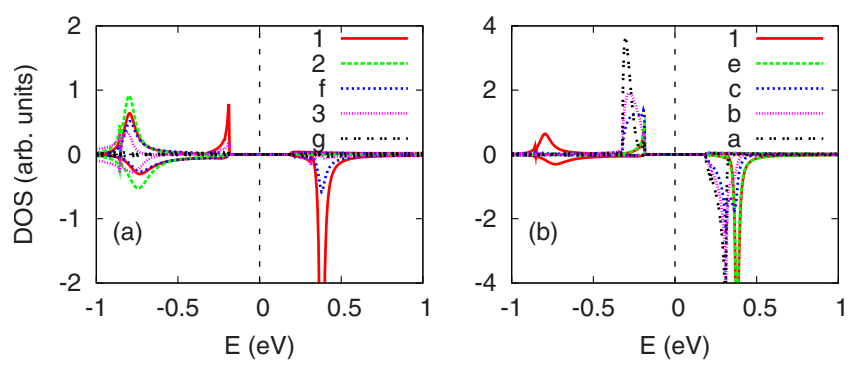

FIG. 7. (Color online) PDOS for atoms located at different sites when one B is doped at site 2: (a) for atoms with increasing distance from $\mathrm{B}$ in the $x$ direction; (b) for atoms with increasing distance from $\mathrm{B}$ on the lower edge.

the impurity atom, although the contribution of B itself is negligible. The magnitude of such states decays rapidly from $\mathrm{B}$ to the ends of the scattering region along the $z$ direction [see Fig. 6]. These states, which correspond to true bound states for $\delta=0$, acquire a very weak coupling to the leads for a finite $\delta$. Therefore the sharp transmission peaks in the energy gap for finite $\delta$ arise from bound states now coupled to the leads by disorder. We can observe an excellent one-toone correspondence between the DOS peaks of the bound states and the transmission peaks in the gap [see Fig. 6]. For example, in Fig. 4(a), only one peak is observed in the gap, while in the other cases, double peaks can be found. The multiplicity of the bound states arises from the different interaction strength between the edge states and the impurity atom. We finally note that, although bound states do not contribute significantly to the transport, they should be detectable in photoemission experiments.

When B is placed at site 2, the PDOS of the edge carbon atoms still decays rapidly from the doping site [see Fig. 7(b)]. Thus the transmission through the edge states remains strongly suppressed. We can again identify quasibound states at about $-0.8 \mathrm{eV}$ [see Fig. 7(a)], localized around the impurity site. The transmission dips at $-0.8 \mathrm{eV}$ arise from these states. When taking into account disorder $\left(\delta=10^{-4} \mathrm{Ry}\right)$, sharp peaks appear in the DOS at $-0.03 \mathrm{eV}$ for the majority spin and at $-0.16 \mathrm{eV}$ for the minority. These bound states are localized mainly on the carbon atoms at the lower and upper edges [see Fig. 8]. When the coupling of these states to the leads is finite, electrons from one lead can tunnel resonantly to the other lead, so that gap resonances are observed in the transmission function [see Fig. 4].
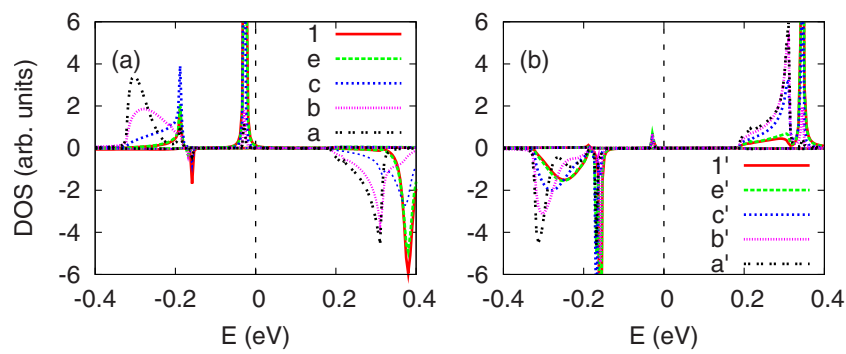

FIG. 8. (Color online) PDOS for atoms located at different sites when doping $\mathrm{B}$ at site 2 , with $\delta=10^{-4} \mathrm{Ry}$ : (a) for atoms with increasing distance from $\mathrm{B}$ on the lower edge; (b) for atoms with increasing distance from $\mathrm{B}$ on the upper edge.
Next we analyze the origin of another interesting finding, namely that doping $\mathrm{B}$ and $\mathrm{N}$ at the same site always causes transmission dips located at opposite energy positions relative to $E_{F} \cdot{ }^{18,27}$ In addition, when the same impurity atom is doped at the edge or inside the ribbon, it results in quasibound states also located at opposite sides relatively to $E_{F}{ }^{26,29}$ Consider first the generic problem of doping B inside a carbon system. Since B has one electron less than C, it will attract an electron from its neighboring atoms becoming a $\mathrm{B}^{-}$ ion. As a consequence, an occupied quasibound state is formed around the B atom (we call this a $\mathrm{B}^{-}$state). Such state necessarily lies below the Fermi level. In contrast, $\mathrm{N}$ doping donates one electron to the system thus forming a $\mathrm{N}^{+}$ ion. The corresponding quasibound state lies above the Fermi level. That behavior is observed for instance in carbon nanotubes. ${ }^{18,27}$ However, the situation is different when one dopes $\mathrm{B}$ or $\mathrm{N}$ at the edge of the nanoribbon, which we believe is due to the peculiar localization properties of its edge states.

\section{Multiple dopants}

When two impurities are present in the cell, more features appear in the transmission as compared to the single dopant case. We first look at the situation where the two impurities interact moderately among each other. This is obtained for instance by placing two B atoms at equal distance from the edges (at doping sites $\mathrm{x} 1$ and $\mathrm{x} 1^{\prime}$ or at $\mathrm{x} 2$ and $\mathrm{x} 2^{\prime}, \ldots$, see Fig. 1) and at a relative distance of $4.92 \AA$ [see Figs. 9(a)-9(d)]. Alternatively, we also look at the case where the two B are doped at opposite edges [see Figs. 9(e)-9(h)], but at an equal distance from the relative edge (e.g., sites 1 and $1^{\prime}, 2$ and $2^{\prime}$, etc.). In both these cases we can still observe transmission dips, whose positions most of the times is similar to the one of single dopants placed at the same sites, i.e., there is little interaction between the impurities. However, in general the shape of the transmission spectrum is less regular than that obtained for single impurity doping due to the interference between the two dopants (these have similar energies).

It can be expected that by increasing the impurities concentration, because of the enhanced impurity-impurity interaction, the nature of the quasibound states will become more complex, and this will reflect in the transmission. Thus, wellshaped transmission dips can be observed only for single impurity doping, or when a large difference exists in the energies of the quasibound states associated to the individual dopants. For instance this is achieved if one dopes B atoms at sites 1 and 4 or at sites $x 1$ and $\times 3^{\prime}$. For these two pairs of dopants we can observe that the single dopant features are recovered in the transmission spectrum [see Figs. 10(a) and $10(\mathrm{~b})]$, since the energies of the quasibound states of the individual impurities are, respectively, well above and well below $E_{F}$.

Another example is provided by $\mathrm{B}$ and $\mathrm{N}$ codoping. Also in this case the impurities are either both placed at an equal distance from the lower edge [Figs. 11(a)-11(d)] or alternatively they are placed at sites with an equal distance from opposite edges, with $\mathrm{B}$ closer to the lower edge and $\mathrm{N}$ to the 

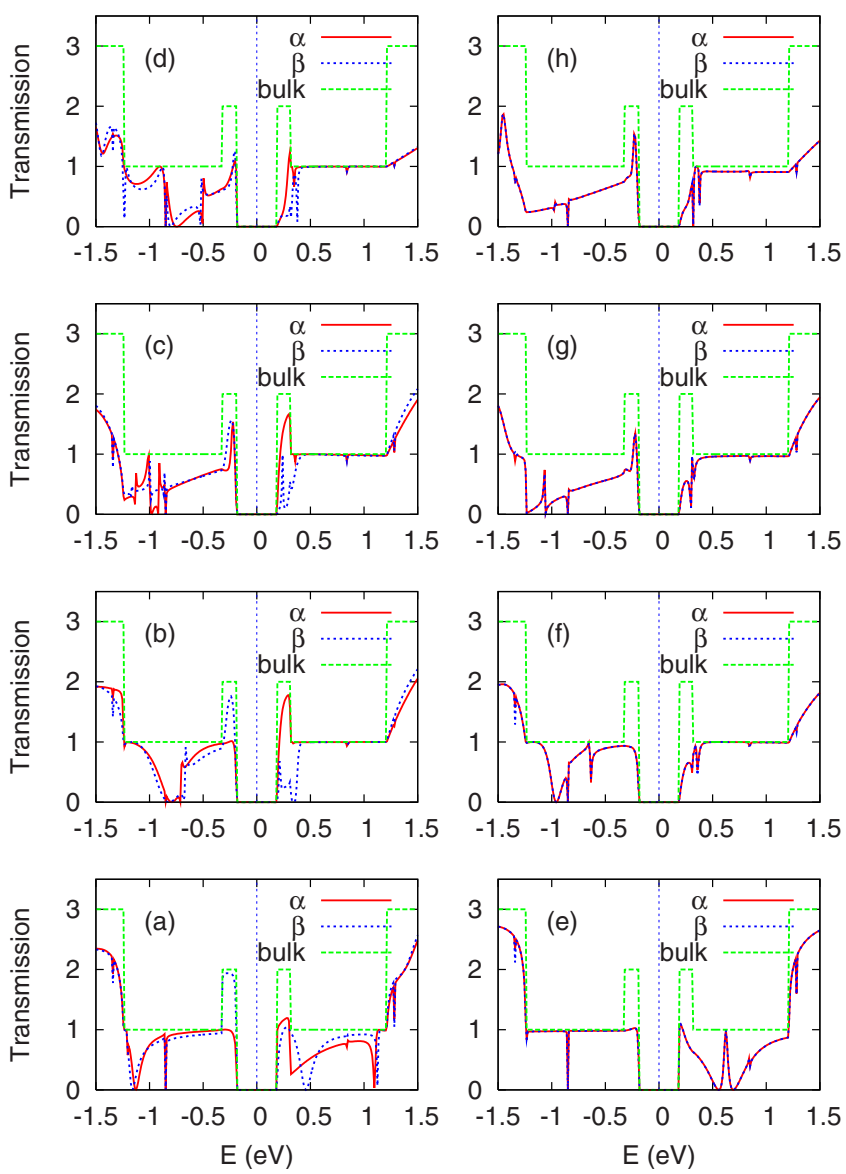

FIG. 9. (Color online) Transmission function when the ribbon is doped with two B atoms. The impurities are either placed on equivalent sites at an equal distance from the lower edge, or at sites with an equal distance, respectively, from the lower and upper edge. The doping sites are: (a) $\mathrm{x} 1$ and $\mathrm{x} 1^{\prime}$; (b) $\mathrm{x} 2$ and $\mathrm{x} 2^{\prime}$; (c) $\mathrm{x} 3$ and $\mathrm{x} 3^{\prime}$ and (d) $x 4$ and $\times 4^{\prime}$; (e) 1 and $1^{\prime}$; (f) 2 and $2^{\prime}$; (g) 3 and $3^{\prime}$; (h) 4 and $4^{\prime}$.

upper one [Figs. 11(e)-11(h)]. The corresponding transmission functions are presented in Fig. 11. In both cases, clear transmission dips, symmetrically located at opposite sides of the Fermi level and induced respectively by B and $\mathrm{N}$ doping, can be observed. Thus, the quasibound states induced by codoping are simply a superposition of those obtained for individual $\mathrm{B}$ and $\mathrm{N}$ doping. The reason for this (see also Fig. 9) is that $\mathrm{N}$-induced quasibound states are always energetically far from the B-induced ones when the doping sites have an equal distance from the edge [see Fig. 2]. Interestingly,
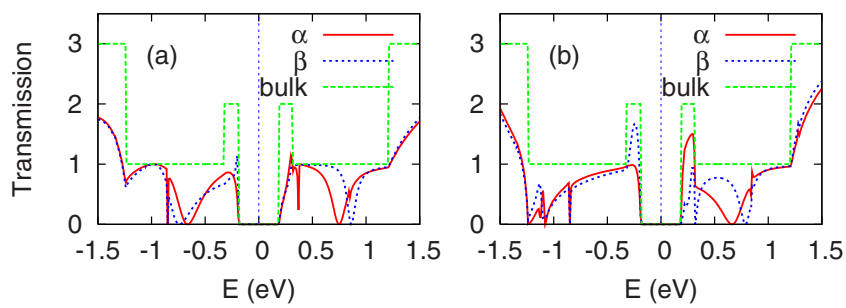

FIG. 10. (Color online) Transmission function when doping two $\mathrm{B}$ atoms at sites: (a) 1 and 4; (b) $\mathrm{x} 1$ and $\mathrm{x} 3^{\prime}$.
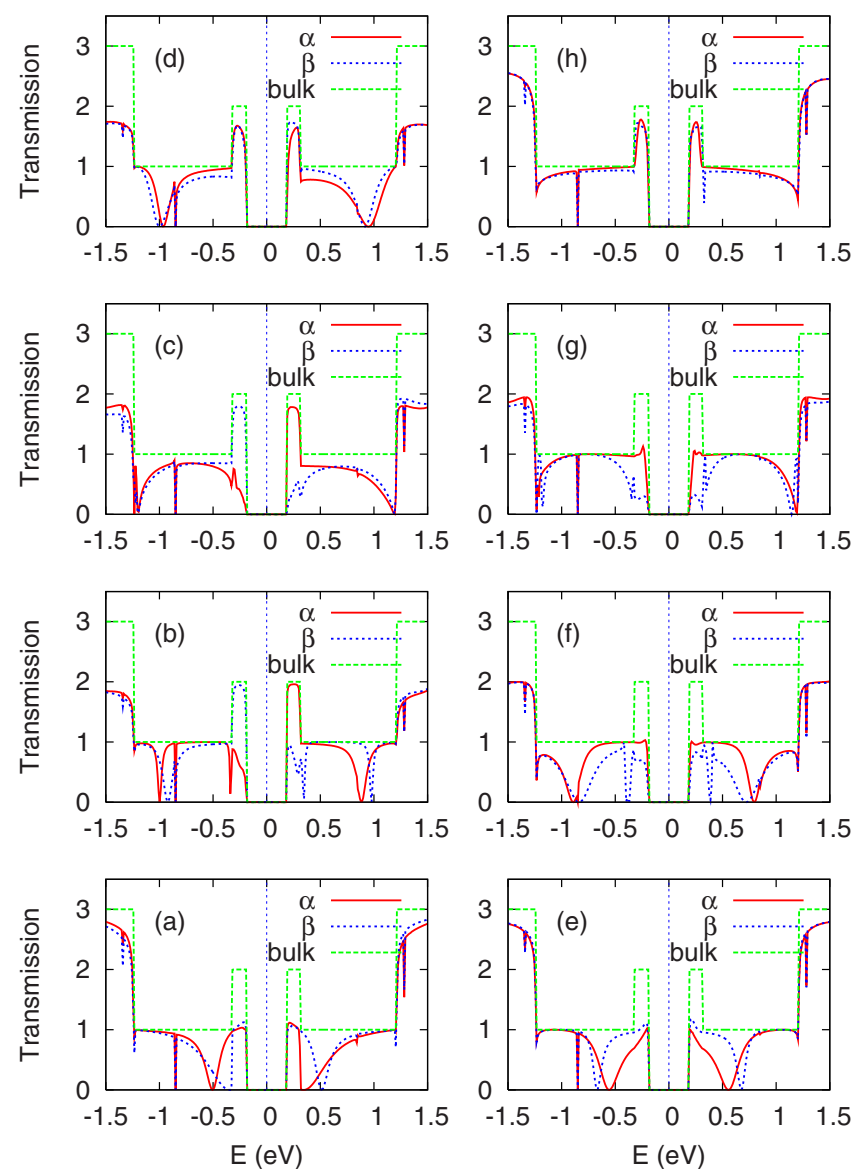

FIG. 11. (Color online) Transmission function for B and N codoping. The impurities are either placed on equivalent sites at an equal distance from the lower edge, or at sites with an equal distance, respectively, from the lower and upper edge. The doping sites are: (a) $\mathrm{x} 1$ and $\mathrm{x} 1^{\prime}$; (b) $\mathrm{x} 2$ and $\mathrm{x} 2^{\prime}$; (c) $\mathrm{x} 3$ and $\mathrm{x} 3^{\prime}$ and (d) $\mathrm{x} 4$ and $\mathrm{x} 4^{\prime}$; (e) 1 and $1^{\prime}$; (f) 2 and $2^{\prime}$; (g) 3 and $3^{\prime}$; (h) 4 and $4^{\prime}$.

the transmission dips practically disappear when the $\mathrm{B}$ and $\mathrm{N}$ dopants are placed, respectively, at sites 4 and $4^{\prime}$, i.e., when they are nearest neighbors. In this situation electron exchange occurs between these two atoms much more prominently than between the impurities and the edge states. For this reason the influence of the doping over the surrounding carbon atoms is much smaller and no quasibound states are formed close to the Fermi energy. This is also the reason of why in the disordered case, which will be discussed next, no bound states are formed in the gap when B and $\mathrm{N}$ are nearest neighbors [see Fig. 12(h)].

If the disorder in the leads is taken into account by setting $\delta=10^{-4} \mathrm{Ry}$, resonant peaks for both the majority and minority spin channels are observed in the gap when the dopants are at an equal distance from the edge [Fig. 12]. These resonant peaks are symmetrically located relatively to the Fermi level. In contrast when $\mathrm{B}$ and $\mathrm{N}$ are doped at an equal distance from opposite edges, the resonant peaks of only one spin channel can be observed in the gap. This gives rise to completely spin polarized electron transport. We argue that such transport behavior through bound states should be experimentally observable in the tunneling conductance of finite nanoribbons attached to metallic electrodes. 

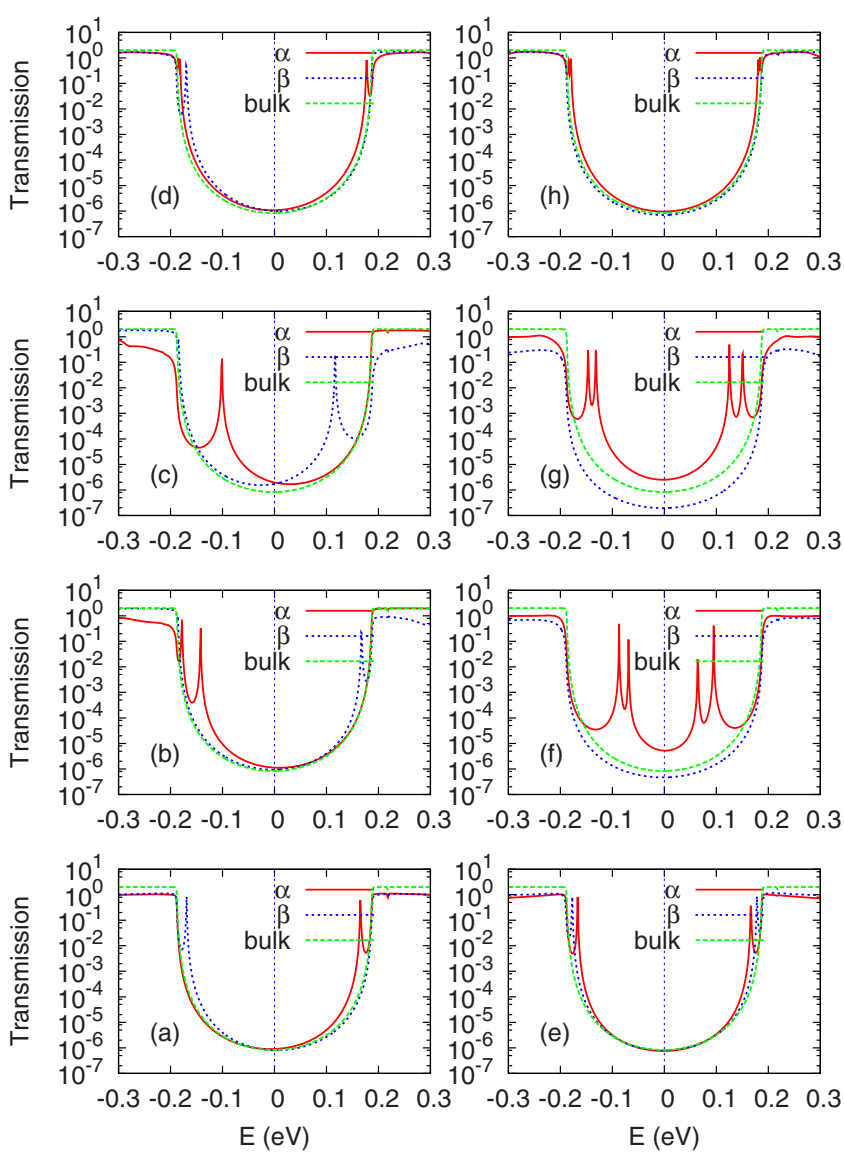

FIG. 12. (Color online) Transmission function for $\mathrm{B}$ and $\mathrm{N}$ codoping in the energy range $[-0.3,0.3] \mathrm{eV}$ for the same doping sites as those of Fig. 11. This time $\delta=10^{-4}$ Ry in the leads.

\section{CONCLUSIONS}

In conclusion, the dependence of the electron transmission function of ZGNRs on the position of $\mathrm{N}$ and $\mathrm{B}$ impurities has been investigated by first principles calculations. It is found that a B/N impurity in the ZGNRs introduces bound states and quasibound states. These greatly modify the extended edge states, resulting in many interesting effects over the transmission. Quasibound states cause wide dips in the transmission function, while bound states induce resonant narrow peaks in the energy gap. These become visible when the coupling between bound states and the leads is finite, as for example when the leads are disordered. When a B or $\mathrm{N}$ impurity is placed close to the edge, the edge states are greatly affected and their associated transmission channel gets suppressed. In particular if the doping site is an edge site, a single impurity alone can completely block the transmission [see, e.g., Figs. 2(a) and 3(a)].

In the case of multiple $\mathrm{B}$ doping, interference between the quasibound states results in a rather complex landscape of dips in the transmission function. However, if the quasibound states induced by the two B atoms or by one B and one $\mathrm{N}$ atom are at opposite sides with respect to the Fermi level, then the features in the transmission function can be approximated as a simple superposition of those originating from single dopants. Finally, when the B and $\mathrm{N}$ atoms are placed at equal positions from different edges, only one spin channel peaks appear in the gap region, which may result in fully spin polarized transport. This mechanism may find applications in spintronic devices.

\section{ACKNOWLEDGMENTS}

This work was supported by the National Science Foundation of China under Grants No. 10774148 and No. 10904148, the Special Funds for Major State Basic Research Project of China (973) under Grant No. 2007CB925004, 863 Project, Knowledge Innovation Program of the Chinese Academy of Sciences, and Director Grants of CASHIPS. Part of the calculations were performed at the Center for Computational Science of CASHIPS and the Shanghai Supercomputer Center, and at the Irish Centre for High End Computing. The SMEAGOL project (I.R. and S.S.) is supported by the Science Foundation of Ireland.

\footnotetext{
*zzeng@theory.issp.ac.cn

${ }^{1}$ K. Wakabayashi, M. Fujita, H. Ajiki, and M. Sigrist, Phys. Rev. B 59, 8271 (1999).

${ }^{2}$ M. Ezawa, Phys. Rev. B 73, 045432 (2006).

${ }^{3}$ D. A. Abanin, P. A. Lee, and L. S. Levitov, Phys. Rev. Lett. 96, 176803 (2006).

${ }^{4}$ S. Okada and A. Oshiyama, Phys. Rev. Lett. 87, 146803 (2001).

${ }^{5}$ Y. W. Son, M. L. Cohen, and S. G. Louie, Nature (London) 444, 347 (2006).

${ }^{6}$ Y. Miyamoto, K. Nakada, and M. Fujita, Phys. Rev. B 59, 9858 (1999).

${ }^{7}$ T. Kawai, Y. Miyamoto, O. Sugino, and Y. Koga, Phys. Rev. B 62, R16349 (2000)

${ }^{8}$ L. Yang, C.-H. Park, Y.-W. Son, M. L. Cohen, and S. G. Louie, Phys. Rev. Lett. 99, 186801 (2007).

${ }^{9}$ K. Nakada, M. Fujita, G. Dresselhaus, and M. S. Dresselhaus,
}

Phys. Rev. B 54, 17954 (1996).

${ }^{10}$ Y. W. Son, M. L. Cohen, and S. G. Louie, Phys. Rev. Lett. 97, 216803 (2006).

${ }^{11}$ V. Barone, O. Hod, and G. E. Scuseria, Nano Lett. 6, 2748 (2006).

${ }^{12}$ S. Reich, J. Maultzsch, C. Thomsen, and P. Ordejon, Phys. Rev. B 66, 035412 (2002).

${ }^{13}$ T. B. Martins, R. H. Miwa, A. J. R. da Silva, and A. Fazzio, Phys. Rev. Lett. 98, 196803 (2007).

${ }^{14}$ E.-J. Kan, Z. Li, J. Yang, and J. G. Hou, J. Am. Chem. Soc. 130, 4224 (2008).

${ }^{15}$ S. Dutta, A. K. Manna, and S. K. Pati, Phys. Rev. Lett. 102, 096601 (2009).

${ }^{16}$ E.-J. Kan, X. J. Wu, Z. Y. Li, X. C. Zeng, J. L. Yang, and J. G. Hou, J. Chem. Phys. 129, 084712 (2008).

${ }^{17}$ J. M. Soler, E. Artacho, J. D. Gale, A. Garcia, J. Junquera, P. 
Ordejon, and D. Sanchez-Portal, J. Phys.: Condens. Matter 14, 2745 (2002).

${ }^{18}$ Y.-W. Son, J. Ihm, M. L. Cohen, S. G. Louie, and H. J. Choi, Phys. Rev. Lett. 95, 216602 (2005).

${ }^{19}$ H. Ren, Q.-X. Li, Y. Luo, and J. L. Yang, Appl. Phys. Lett. 94, 173110 (2009).

${ }^{20}$ J. P. Perdew and A. Zunger, Phys. Rev. B 23, 5048 (1981).

${ }^{21}$ A. R. Rocha, V. Garcia-Suarez, S. W. Bailey, C. J. Lambert, J. Ferrer, and S. Sanvito, Nature Mater. 4, 335 (2005).

${ }^{22}$ A. R. Rocha, V. Garcia-Suarez, S. W. Bailey, C. J. Lambert, J. Ferrer, and S. Sanvito, Phys. Rev. B 73, 085414 (2006).

${ }^{23}$ I. Rungger and S. Sanvito, Phys. Rev. B 78, 035407 (2008).

${ }^{24}$ S. Datta, in Electronic Transport in Mesoscopic Systems, edited by H. Ahmed, M. Pepper, and A. Broers (Cambridge University Press, Cambridge, England, 1995).

${ }^{25}$ H. Haug and A.-P. Jauho, Quantum Kinetics in Transport and Optics of Semiconductors (Springer-Verlag, Berlin, 1996).

${ }^{26}$ B. Biel, X. Blase, F. Triozon, and S. Roche, Phys. Rev. Lett. 102, 096803 (2009).

${ }^{27}$ H. J. Choi, J. Ihm, S. G. Louie, and M. L. Cohen, Phys. Rev. Lett. 84, 2917 (2000)

${ }^{28}$ B. Huang, F. Liu, J. Wu, B.-L. Gu, and W. Duan, Phys. Rev. B 77, 153411 (2008).

${ }^{29}$ S. S. Yu, W. T. Zheng, W. B. Wen, and Q. Jiang, Carbon 46, 537 (2008). 